\title{
Leonhard Euler (1707-1783)
}

\section{GRAHAM HOARE}

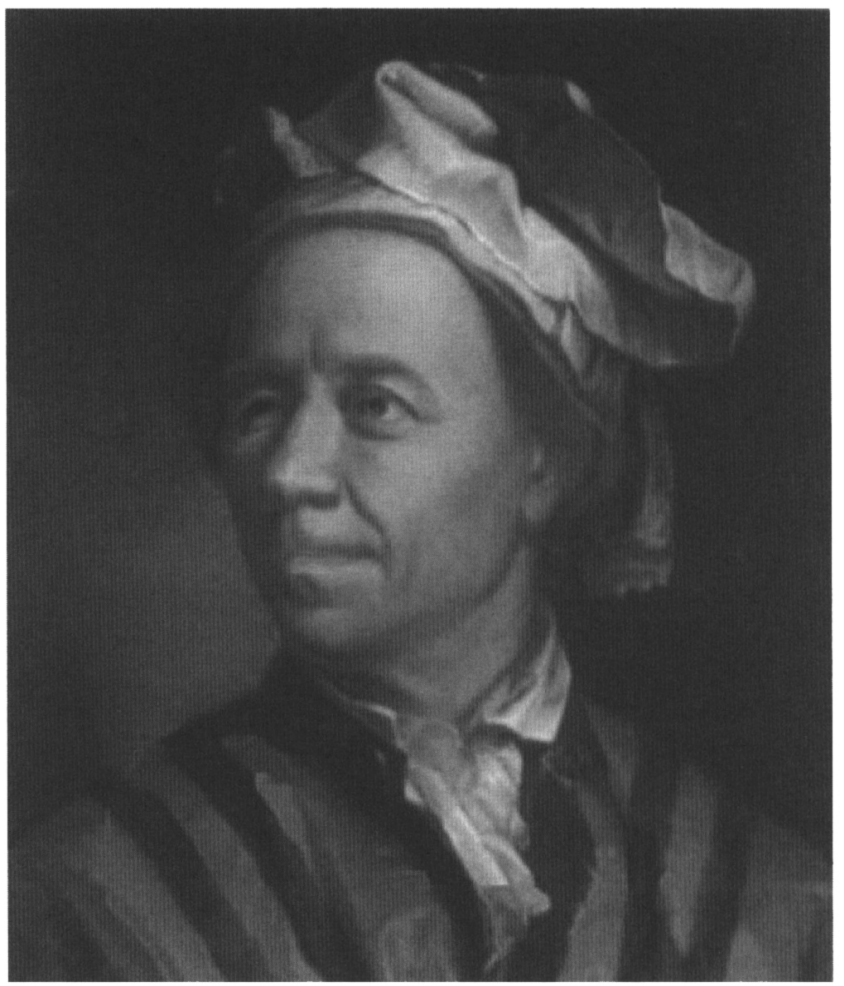

Life

In the preamble to his lecture to the International Congress of Mathematics at Paris in 1900, in which he posed his now-famous 23 problems, David Hilbert said that, "As long as a branch of science offers an abundance of problems, so long is it alive; ... . It is by the solution of problems that the investigator tests the temper of his steel; he finds new outlooks, and gains a wider and freer horizon.' By these criteria Leonhard Euler, reputedly the most prolific mathematician of all time, must be judged one of the greatest masters of the mathematical arts. The scope and versatility of his thinking were prodigious. Indeed, we can discern his hand in three of the seven Millennium Problems announced in Paris in May 2000 which have a classical mathematical basis. The departure point for Riemann's work on the Riemann Hypothesis is the zeta function whose number-theoretic properties were first established by Euler. Euler's equations for the motion of a frictionless incompressible fluid as well as their extensions to allow for viscosity, the Navier-Stokes equations, continue 
to challenge mathematicians for solutions. The Poincaré Conjecture, ostensibly resolved in 2002 by Grigori Perelman, involves the classification of manifolds. One of the distinguishing topological invariants for such objects is Euler's characteristic, $V-E+F$, generalised by Poincaré to higher dimensions.

Euler was born in Basel, Switzerland, on 15 April 1707. His father, Paul, was a Protestant minister who had attended Johann Bernoulli's lectures in mathematics at the University of Basel. Before attending school Euler was tutored by his father in a number of subjects but, undoubtedly, it was the instruction he received in elementary mathematics that sparked his enthusiasm for the subject. School involved attendance at a mediocre Gymnasium and to remedy the absence of mathematics from the curriculum the young Euler studied privately with Johann Burckhardt, an amateur mathematician, and read such texts as Christoff Rudolf's Algebra. However, we shall see that Euler's destiny as a mathematician and the trajectory of his life were linked, directly or indirectly, to the Bernoulli dynasty.

In the autumn of 1720 , at the age of 13, Euler enrolled in the department of Arts at the University of Basel to receive a general education before specialising. At this time Johann Bernoulli was professor of mathematics having succeeded his brother Jakob late in 1705 in the chair. According to his unpublished biographical writings Euler, dissatisfied with his studies, engineered an introduction to 'a famous professor, Johann Bernoulli'. The professor was very busy and refused to give Euler private lessons but advised him to begin reading advanced mathematical texts. It was arranged for Euler to visit the professor on Saturday afternoons when he would receive explanations for 'everything I could not understand'. In 1723 Euler completed his Master's degree in philosophy with a dissertation comparing the philosophical ideas of Descartes and Newton. In accordance with his father's wishes Euler now began studying theology in the autumn of 1723 with a view to following him in the ministry. However, he could not summon any great enthusiasm for his theological studies and devoted most of his time to mathematics. It was here that Johann Bernoulli intervened to persuade Euler's father to allow his son to switch to mathematics. Johann had become increasingly aware that Euler was a mathematical genius in the making.

In 1726 Euler completed his formal studies. Advised by Bernoulli, he had studied the works of, amongst others, Descartes, Galileo, Newton, Jakob Bernoulli, Taylor, Wallis and Jakob Hermann, a distant relative. By the age of 18 he began his own independent investigations and his first paper, a brief note on the construction of isochronous curves in a resisting medium, appeared in 1726 . This was followed in 1727 by an article on algebraic reciprocal trajectories. But now Euler sought an academic position. Switzerland was no stamping ground for aspiring mathematicians; there were few publications and few openings for academics. He applied for the chair of Physics at Basel university on the death of the incumbent but the decision to reject him was propitious. 
In the autumn of 1725 Johann Bernoulli's sons, Nikolaus and Daniel, went to Russia. Nikolaus, however, died in St Petersburg in July 1726 thereby creating a vacancy which was offered to Euler on the recommendation of the Bernoullis. Euler accepted the post in November 1726 but delayed his departure until April 1727 as he wished to ascertain the outcome of his application for the Physics post, mentioned earlier, and to study anatomy and physiology. His first appointment at the newly created $S t$ Petersburg Academy of Sciences required him to teach the applications of mathematics and mechanics to physiology. Having arrived in St Petersburg in May 1727 Euler was soon transferred to the mathematical-physical division of the Academy at the behest of Daniel Bernoulli and Jakob Hermann. From this time his life and work were associated with St Petersburg and Russia. He was surrounded by a group of eminent scientists who contributed to the flowering of his genius. There was Jakob Hermann, geometer and analyst, Daniel Bernoulli, a personal friend, with whom he shared common interests in applied mathematics, Christian Goldbach, with whom he discussed problems in analysis and number theory, F. Maier, expert in trigonometry and the astronomer and geographer J-N Delisle. In a letter dated 1749 Euler cited that he and others owed everything to the favourable conditions that prevailed at the Academy. In 1730 Euler was appointed professor of Physics. Then, three years later, Daniel Bernoulli decided to return to Basel and Euler succeeded him as the premier mathematician at the Academy. In 1734, his finances now secure, Euler married Katharina Gsell, the daughter of a Swiss artist then working in Russia. Of the thirteen children they had together only five survived infancy. Euler would claim that he could continue his mathematical work while his offspring clambered over him!

During the fourteen years he was to spend in the first of two sojourns in St Petersburg, Euler made brilliant discoveries in such areas as analysis, number theory, differential equations, the calculus of variations and mechanics. By 1741 he had prepared some 90 works for publication. 55 of these appeared in print including the two-volume Mechanica in which he systematically applied mathematical analysis to Newtonian dynamics. We should note, too, that Euler formulated many of his principal ideas and creative concepts when he was quite young. He had studied hydrodynamics in Basel but his most important memoirs on the subject didn't appear until the mid-1750s. Ideas he had in the 1730s of a systematic exposition of the differential calculus based on finite differences only came to fruition some 20 years later. Euler's copious correspondence ensured that his nascent ideas and discoveries were well known before their publication. As well as his commitments in mathematics Euler was in charge of the Geography department. This involved producing maps in collaboration with Delisle. Euler was to attribute the loss of his right eye in 1738 to strain caused by his cartographic work but the problem may have begun earlier in 1735 when he suffered a severe fever which almost ended his life.

Having won the Grand Prix of the Paris Academy in 1738 and 1740 Euler's pre-eminence was assured and he looked settled in St Petersburg. 
However, Catherine I died suddenly and the Russian government passed into the hands of those who regarded a scientific academy with suspicion. Meanwhile, Frederick the Great having succeeded to the Prussian throne in June 1740, decided to reorganise the Berlin Society of Sciences which had been founded by Leibniz. Aware of Euler's reputation, the King invited him to Berlin. An increasingly isolated Euler accepted and arrived there in 1741 . He was appointed director of the mathematical section of the newly founded Berlin Academy in 1744 and was free to choose his own research programme. Euler undertook numerous other duties which included supervising the observatory and botanical gardens, selecting personnel, superintending financial matters and managing the publication of calendars and maps which provided a source of income for the Academy. Correspondence with professors at German universities entailed that the considerable influence he wielded at the Academy was far from localised. Furthermore, apart from a period when there were hostilities between Russia and Prussia, Euler maintained contact with the St Petersburg Academy during the 25 years he was in Berlin and received a full pension from his previous employers. In return he purchased books and instruments for the Academy and wrote scientific reports for them.

After its president, Pierre Maupertius, died in 1759 the Berlin Academy was managed by Euler under direct supervision of the King. When Frederick offered the presidency to D'Alembert, Euler's rival in scientific matters, Euler became unsettled. The King had little appreciation of higher mathematics and perceived science as the servant of the state and as a vehicle for technological progress. Although D'Alembert refused to move to Berlin it was because of Frederick's continued interference in such matters as finances and the making of appointments that Euler, having enjoyed considerable independence hitherto, decided that it was time to leave. Reciprocally, Euler had fallen out of favour with the King who had little time for those of a humble disposition. Even so the King was furious when his 'cyclops' left Berlin in 1766. Euler's return to St Petersburg had been instigated by Catherine the Great who subsequently treated him as visiting royalty.

Euler's mathematical and scientific output during his time in Berlin was truly astonishing. In all he prepared 380 works, 275 of which were published including 7 lengthy books. Competing with D'Alembert and Daniel Bernoulli he laid the foundations of mathematical physics and was a rival of A. Clairaut and D'Alembert in advancing the theory of lunar and planetary motion. He also elaborated the theory of motion of solids, developed a systematic treatment of hydrodynamics and of the differential geometry of surfaces. His 1744 masterpiece, the memoir on the calculus of variations, earned his election to the Royal Society of London and to the Paris Academy. In 1750 he conjectured the $V-E+F=2$ formula for convex polyhedra and attempted to prove it. In a more popular vein he wrote the celebrated Letters to a German Princess, the King's niece, in the period 1760-62. These 234 letters presented the most comprehensive and 
authoritative exposition on natural philosophy by a leading scientist of the eighteenth century. Euler even found time to produce works on ballistics, shipbuilding and navigation.

Soon after his return to St Petersburg, Euler was beset by misfortune. Following a brief illness he became almost completely blind; he could no longer read. Then in 1771 his home on the embankment of the river Neva burnt down but he was able to save his mathematical manuscripts. Shortly after a cataract operation had restored his sight temporarily total blindness ensued. In 1773 Euler's wife died and 3 years later he married her half sister, Salome Gsell.

Possessed of a flawless memory (he knew Virgil's Aeneid by heart), Euler, amazingly, produced almost half of his works during his second stint in St Petersburg. In these endeavours he was helped by his sons Johann and Christophe and by the academician J. Lexell and his disciple N. Fuss. He continued his work on optics, algebra and lunar motion. His Algebra (1770) was dictated to his valet and became the most successful mathematics textbook since Euclid's Elements.

Euler spent the first half of the last day of his life, 18 September 1783, as usual. He gave mathematics tuition to one of his grandchildren, chalked some calculations on two boards on the motion of balloons and then discussed the motion of the recently discovered planet Uranus with Lexell and Fuss. At five o'clock he suffered a brain haemorrhage, announced 'I am dying', before losing consciousness, and died at about eleven o'clock (see [1]). His grave can be located in the 18th century necropolis close to the Alexander Nevsky Monastery. A massive monument atop the grave bears the inscription 'Leonhardo Eulero Academia Petropolitana'. For nearly 50 years after his death the Academy at St Petersburg continued to publish Euler's works.

Of Euler's many admirable qualities his willingness to explain how he made his discoveries was paramount. He was unique in revealing his preliminary guesses, experiments and partial proofs. Readers will be familiar with Chapter 6 of G. Pólya's book [2] that includes a translation of the memoir in which Euler announced the pentagonal number theorem.

\section{Mathematics}

Much of the Euler canon concerned applications of mathematics but twenty-nine volumes of the Opera omnia were devoted to Pure mathematics. Seventeen of these involved analysis, a subject which required serious attention at the time. Newton's presentation of the Calculus, for example, was controversial for his arguments deployed infinitesimals and fluxions which some, especially Bishop Berkeley, rightly considered contradictory. Euler's efforts to systematise analysis were mostly successful but his calculus of zeros, designed to give differential calculus a secure foundation, though sound, was inadequate for further development; analysis eventually would be grounded in the notion that infinitesimals are variables 
tending to zero in the limit. By contrast, his researches into and invention of methods of indefinite integration, where he treats exhaustively those cases that can be expressed by elementary functions, are impressive by today's standards.

In the Introductio in analysis infinitorum (1748) Euler presented the first clear notion of a function as a correspondence of values. He perceived mathematical analysis as a science of functions which are produced by algebraic, elementary and transcendental operations facilitated by integration. For the most part the functions studied by Euler and other mathematicians of the eighteenth century were analytic and Euler, accordingly, exploited power series to express them. Euler's constant, $\gamma$, was introduced in the context of infinite series where he showed that it was the limit of $\sum_{1}^{n} \frac{1}{r}-\ln n$ as $n$ tends to infinity. He was the first to express an algebraic function by a Fourier series when he gave the result

$$
\frac{\pi}{2}-\frac{x}{2}=\sin x+\frac{\sin 2 x}{2}+\frac{\sin 3 x}{3}+\cdots
$$

Euler also introduced the beta- and gamma- functions and derived some of their properties. The theory of multiple integration emanated from him and he established the theorem for homogeneous functions, namely, if $f(x, y)$ satisfies $f(\lambda x, \lambda y)=\lambda^{n} f(x, y)$, then $x \frac{\partial f}{\partial x}+y \frac{\partial f}{\partial y}=n f$. A case in point where Euler's work was motivated by applications was his investigation of elliptic functions which began with his study of elastica, a curve described by a thin elastic rod compressed at the ends, which was discovered by Jakob Bernoulli. Euler's paper of 1757 , in which he proved the famous addition theorem for elliptic integrals, established them as a subject of independent interest.

Euler's excursions into the realm of functions of complex variables are well known. In the Introductio Euler firstly described the analytic theory of trigonometric functions for the first time and followed this with a thoroughgoing presentation of both plane and solid analytical geometry. Here, he deduced, non-rigorously, the formula $e^{i x}=\cos x+i \sin x$ which yields $e^{i \pi}+1=0$, a result rated in recent surveys as the most beautiful in mathematics (see [3]). Euler investigated analytic functions of a complex variable in a variety of contexts. These studies led him to conclude that complex numbers are closed under algebraic and transcendental operations. In 1777 he deduced the Cauchy-Riemann equations, $\frac{\partial u}{\partial x}=\frac{\partial v}{\partial y}, \frac{\partial u}{\partial y}=-\frac{\partial v}{\partial x}$, connecting the real and complex parts of the analytic function $u(x, y)+i v(x, y)$, although d'Alembert had discovered them earlier whilst researching problems in hydrodynamics. Euler also exploited complex variable substitutions to resolve such integrals as

$$
\int_{0}^{\infty} \frac{\sin x}{x} d x\left(=\frac{\pi}{2}\right)
$$

Euler was led to a wide-ranging study of ordinary and partial 
differential equations in his work in mathematical physics. His technique for solving linear equations with constant coefficients carefully distinguished between general and particular integrals. He also considered second order differential equations with variable coefficients, power series solutions, the concept of an integrating factor and he created the method for approximating the solution of the equation $\frac{d y}{d x}=f(x, y)$, with initial condition $x=x_{0}, y=y_{0}$, extending it to second-order equations. His work on vibrating membranes led him to the Bessel equations which he solved by means of Bessel functions.

Stimulated by the Bernoullis and Goldbach and challenged by a number of unproven conjectures of Fermat, Euler laid the foundations of number theory as an autonomous discipline. He soon demolished Fermat's conjecture that the numbers $2^{2^{n}}+1$ were prime when he found that $2^{32}+1$ had a factor of 641 . He proved Fermat's Little Theorem by three methods, the third of which he generalised to $a^{\phi(n)}-1=0(\bmod n)$, where $a, n$ are coprime and $\phi(n)$ denotes Euler's function which counts the number of positive integers less than and coprime to $n$. He also proved that $a^{2}+b^{2}$, where $a, b$ are coprime, has no divisor of the form $4 n-1$. He contributed to Diophantine analysis when, for example, he gave a method for calculating the smallest solution of the equation $x^{2}-d y^{2}=1(d>0$, non-square $)$ using continued fractions. His proof of the impossibility of solving $x^{3}+y^{3}=z^{3}$, a particular case of Fermat's Last Theorem, exploited infinite descent but he chose also to employ complex numbers of the form $a+b \sqrt{-3}$ which he assumed to be uniquely factorisable, an unfounded but correct assumption. As it happened Euler had all the necessary machinery to fashion a sound proof. Euler pioneered the use of analytical methods in number theory. Using the expansions of certain infinite products into power series whose coefficients give the solution, Euler solved the partition problem to find the total number, $p(n)$, of ways the positive integer $n$ can be expressed as a sum of positive integers. He gave the generating function for

$$
\begin{aligned}
p(n) \text { as } \prod_{r=1}^{\infty}\left(1-x^{r}\right)^{-1} & =1+\sum_{n=1}^{\infty} p(n) x^{n}, \text { where } \\
& \prod_{r=1}^{\infty}\left(1-x^{r}\right)=1+\sum_{n=1}^{\infty}(-1)^{n}\left(x^{\left(3 n^{2}+n\right) / 2}+x^{\left(3 n^{2}-n\right) / 2}\right)
\end{aligned}
$$

is known as Euler's formula. From these the beautiful identity

$$
p(n)-p(n-1)-p(n-2)+p(n-5)+p(n-7)-p(n-12)-p(n-15)+\ldots=0
$$

can be derived and a similar one for the sum of the divisors of a number. In Riemann's seminal paper of 1859 , in which he states the Riemann Hypothesis, his starting point is Euler's product formula

$$
\zeta(s)=\sum_{n} \frac{1}{n^{s}}=\prod_{p}\left(1-p^{-s}\right)^{-1}
$$

( $n \in \mathbb{Z}^{+}, p$ prime). Using the summation of divergent series and induction 
Euler discovered the functional equation,

$$
\pi^{-s / 2} \Gamma\left(\frac{s}{2}\right) \zeta(s)=\pi^{-(1-s) / 2} \Gamma\left(\frac{1-s}{2}\right) \zeta(1-s),
$$

which Riemann rediscovered having extended the scope of $s$ to the domain of complex numbers (see [3]). Perhaps the result that brought Euler early fame was his successful rendering of $\zeta(2)$ in closed form, namely $\frac{\pi^{2}}{6}$, a problem that had defeated the Bernoulli brothers, Leibniz, Stirling and de Moivre among others. He developed the generalisation, $\zeta(2 n)=C \pi^{2 n}$ and eventually expressed the rational coefficients $C$ in terms of Bernoulli numbers.

In 1736 Euler outlined an ambitious programme of studies of mechanics in his Mechanica. His methods in this field were distinguished by the systematic application of analysis, unlike the work of his predecessors which were piecemeal. His first efforts analysed the kinematics and dynamics of a point mass both in a vacuum and in a resisting medium. Euler's analysis of the motion of a point mass under a central force constitutes a brilliant reworking of Newton's consideration of the same problem in the Principia and served as an introduction to his further studies in celestial mechanics. It was this work which won him many prizes from the Paris Academy of Sciences. In his consideration of the motion of a point mass on a surface Euler had to solve various problems of differential geometry and geodesics.

In his 1765 publication of Theoria motus corporum solidorum Euler showed that the instantaneous motion of a solid body was reducible to translational and rotational components. He devised differential equations referred to the principal axes of inertia for the motion. In particular, Euler solved the problem of the motion of a solid body about a fixed point where the centre of mass coincides with the fixed point. Solutions of this kind of problem were expressible in terms of elliptic integrals (see [4]). In his 1744 thesis, Methodus inveniendi lineas curvas maximi minimive proprietate gandentes, on the calculus of variations, Euler first introduced the principle of least action which asserts that among all curves joining two points in a plane, a particle follows the curve which minimises the integral $\int m v d s$. In general the integral $\int f\left(x, y, y^{\prime}\right) d x$, where $y^{\prime}=\frac{d y}{d x}$ has a stationary value if the Euler-Lagrange differential equation $\frac{\partial f}{\partial y}-\frac{d}{d x}\left(\frac{\partial f}{\partial y^{\prime}}\right)=0$ is satisfied. Euler noticed that the variational equations remained valid irrespective of the coordinate variables employed. Thus he explicitly recognised in a particular mathematical context that calculus is essentially analytical in character and not tied to any geometrical interpretation conferred upon the symbolism.

Many of the symbols in current use are attributable to Euler. For example, $e$ and $i$, so brilliantly combined with $\pi$ in one succinct relation, we owe to him. He also initiated the use of the letter $f$ and of parentheses for a function, viz $f\left(\left[\frac{x}{a}\right]+c\right)$, the familiar signs for trigonometric functions, the notations $\Delta y, \Delta^{2} y, \ldots$ for finite differences and $\Sigma$ for summation. 
Euler has been criticised for the cavalier manner in which he manipulated mathematical objects, especially infinite series, without due regard to rigour. His judgments, however, were born of a profound intuition and were uncannily unerring. Most of his results that were established somewhat dubiously were eventually tightened up by those who followed him in the nineteenth and twentieth centuries. The progress of calculus was hardly hindered by foundational problems in the eighteenth century. The emergence of the concept of a function led mathematicians away from geometrical objects to considerations of classifying and solving differential or partial differential equations and eventually, for example, to the calculus of variations which Euler created as a branch of mathematics almost singlehandedly. Euler did not abandon the notion of a differential but his treatment of this concept prompted the idea of functionality in his 'differential' calculus. The charge that Euler's thinking is primarily algorithmic can be met, too. He did seek direct solutions to a wide range of practical problems in the sciences, technology and public life and he often prolonged his researches until he had devised a suitable formula for calculation or a numerical solution or even a table. More importantly, these investigations involved a rich panoply of original techniques and methods. Euler's legacy is the influence he had on the work of many distinguished nineteenth century mathematicians. Save for Lagrange, Euler was unrivalled in the eighteenth century and is now generally regarded as one of the most remarkable creators of mathematics since Newton.

This article is dedicated to the memory of the late Gertraude Ober. Although she was far from well she insisted we visit St Petersburg. Our time there in early October 2005 was enhanced by superb weather and beautiful autumnal colours. We chanced upon the tomb of Euler in the 18th century necropolis of the Lazarevskoye cemetery and noticed two freshly cut red carnations lying alongside. We discovered that a professor at the University of St Petersburg leads his recently graduated students of mathematics to the necropolis to pay homage to Euler in time-honoured manner. The carnations can be seen in the cover picture.

\section{References}

1. A. P. Youschkevitch, Dictionary of scientific biography, New York (1970-1990).

2. G. Pólya, Introduction and analogy in mathematics, Princeton (1954).

3. Graham Hoare, More mathematical reflections, Mathematics Today, 41 (June 2005) pp. 87-90.

4. Graham Hoare, Euler and elliptic integrals, Mathematics Today, $\mathbf{4 2}$ (August 2006) pp. 135-138.

GRAHAM HOARE

3 Russett Hill, Chalfont St Peter SL9 8JY 